\title{
Measurements of Diffractive Processes at CDF
}

\author{
KONSTANTIN GOULIANOS (for the CDF Collaboration) \\ The Rockefeller University, New York, NY 10023, USA \\ Presented at the $14^{\text {th }}$ Topical Conference on Hadron Collider Physics, \\ HCP-2002, Karlsruhe, Germany, 29 Sep - 4 Oct 2002.
}

Summary. We review the results of measurements on hard diffractive processes performed by the CDF Collaboration and report preliminary CDF results on two soft diffractive processes with a leading antiptoton and a rapidity gap in addition to that associated with the antiproton. All results have been obtained from data collected in Run I of the Fermilab Tevatron $\bar{p} p$ collider.

\section{Introduction}

Diffractive $\bar{p} p$ interactions are characterized by a leading (high longitudinal momentum) outgoing proton or antiproton and/or a large rapidity gap, defined as a region of pseudorapidity, $\eta \equiv-\ln \tan \frac{\theta}{2}$, devoid of particles. The large rapidity gap is presumed to be due to the exchange of a Pomeron, which carries the internal quantum numbers of the vacuum. Rapidity gaps formed by multiplicity fluctuations in non-diffractive (ND) events are exponentially suppressed with increasing $\Delta \eta$, so that gaps of $\Delta \eta>3$ are mainly diffractive. At high energies, where the available rapidity space is large, diffractive events may have more than one large gap.

Diffractive events that incorporate a hard scattering are referred to as hard diffraction. In this paper we review briefly the results on hard diffraction published by CDF and present preliminary results on two types of soft diffraction events with two diffractive rapidity gaps in an event, as shown schematically in Fig. 1.

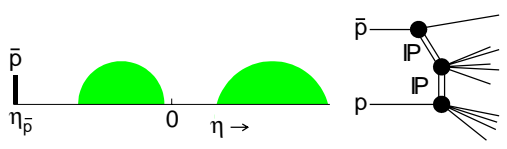

(a) SDD

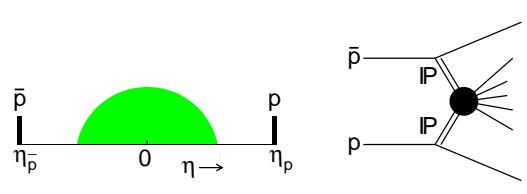

(b) DPE

Fig. 1. Schematic drawings of pseudorapidity topologies and Pomeron exchange diagrams for (a) single plus double diffraction and (b) double Pomeron exchange. 


\section{Hard diffraction}

The CDF results on hard diffraction fall into two classes, characterized by the signature used to identify and extract the diffractive signal: a large rapidity gap or a leading antiproton.

\subsection{Rapidity gap results}

Using the rapidity gap signature to identify diffractive events, CDF measured the single-diffractive (SD) fractions of $W$ [1], dijet [2], $b$-quark [3] and $J / \psi[4]$ production in $\bar{p} p$ collisions at $\sqrt{s}=1800 \mathrm{GeV}$ and the fraction of dijet events with a rapidity gap between jets (double-diffraction - DD) at $\sqrt{s}=1800[8]$ and $630[9] \mathrm{GeV}$. The results for the measured fractions are shown in Table 2.1.

Table 1. Diffractive fractions

\begin{tabular}{|c|c|c|c|}
\hline Hard process & $\sqrt{s}(\mathrm{GeV})$ & $R=\frac{\mathrm{DIFF}}{\text { TOTAL }}(\%)$ & Kinematic region \\
\hline \multicolumn{4}{|l|}{ SD } \\
\hline$W(\rightarrow e \nu)+\mathrm{G}$ & 1800 & $1.15 \pm 0.55$ & $E_{T}^{e}, \not_{T}>20 \mathrm{GeV}$ \\
\hline Jet+Jet+G & 1800 & $0.75 \pm 0.1$ & $E_{T}^{j e t}>20 \mathrm{GeV}, \eta^{j e t}>1.8$ \\
\hline$b(\rightarrow e+X)+\mathrm{G}$ & 1800 & $0.62 \pm 0.25$ & $\left|\eta^{e}\right|<1.1, p_{T}^{e}>9.5 \mathrm{GeV}$ \\
\hline$J / \psi(\rightarrow \mu \mu)+\mathrm{G}$ & 1800 & $1.45 \pm 0.25$ & $\left|\eta^{\mu}\right|<0.6, p_{T}^{\mu}>2 \mathrm{GeV}$ \\
\hline DD & & & \\
\hline Jet-G-Jet & 1800 & $1.13 \pm 0.16$ & $E_{T}^{j e t}>20 \mathrm{GeV}, \eta^{j e t}>1.8$ \\
\hline Jet-G-Jet & 630 & $2.7 \pm 0.9$ & $E_{T}^{j e t}>8 \mathrm{GeV}, \eta^{\text {jet }}>1.8$ \\
\hline
\end{tabular}

Since the different SD processes studied have different sensitivities to the gluon/quark ratio of the interacting partons, the approximate equality of the SD fractions at $\sqrt{s}=1800 \mathrm{GeV}$ indicates that the gluon fraction of the diffractive structure fraction of the proton (gluon fraction of the Pomeron) is not very different from the proton's inclusive gluon fraction. By comparing the fractions of $W, J J$ and $b$ production with Monte Carlo predictions, the gluon fraction of the Pomeron was found to be $f_{g}=0.54_{-0.14}^{+0.16}$ [3]. This result was confirmed by a comparison of the diffractive structure functions obtained from studies of $J / \psi$ and $J J$ production, which yielded a gluon fraction of $f_{g}^{D}=0.59 \pm 0.15[4]$.

\subsection{Leading antiproton results}

Using a Roman pot spectrometer to detect leading antiprotons and determine their momentum and polar angle (hence the $t$-value), CDF measured the ratio of SD to ND dijet production rates at $\sqrt{s}=630$ [6] and $1800 \mathrm{GeV}$ [5] 
as a function of $x$-Bjorken of the struck parton in the $\bar{p}$. In leading order QCD, this ratio is equal to the ratio of the corresponding structure functions. For dijet production, the relevant structure function is the color-weighted combination of gluon and quark terms given by $F_{j j}(x)=x\left[g(x)+\frac{4}{9} \sum_{i} q_{i}(x)\right]$. The diffractive structure function, $\tilde{F}_{j j}^{D}(\beta)$, where $\beta=x / \xi$ is the momentum fraction of the Pomeron's struck parton, is obtained by multiplying the ratio of rates by the known $F_{j j}^{N D}$ and changing variables from $x$ to $\beta$ using $x \rightarrow \beta \xi$ (the tilde over the $F$ indicates integration over $t$ and $\xi$ ).

The $\mathrm{CDF} \tilde{F}_{j j}^{D}(\beta)$ is presented in Fig. 2a and compared with a calculation based on diffractive parton densities obtained by the $\mathrm{H} 1$ Collaboration at HERA from a QCD fit to diffractive DIS data. The CDF result is suppressed by a factor of $\sim 10$ relative to the prediction from from HERA data, indicating a breakdown of factorization of approximately the same magnitude as that observed in the rapidity gap data.

Factorization was also tested within $C D F$ data by comparing the ratio of $\mathrm{DPE} / \mathrm{SD}$ to that of SD/ND dijet production rates (Fig. 2b). The DPE events were extracted from the leading antiproton data by requiring a rapidity gap in the forward detectors on the proton side. At $\langle\xi\rangle=0.02$ and $\left\langle x_{b j}\right\rangle=0.005$, the ratio of $\mathrm{SD} / \mathrm{ND}$ to $\mathrm{DPE} / \mathrm{SD}$ rates normalized per unit $\xi$ was found to be [7] $0.19 \pm 0.07$, violating factorization.
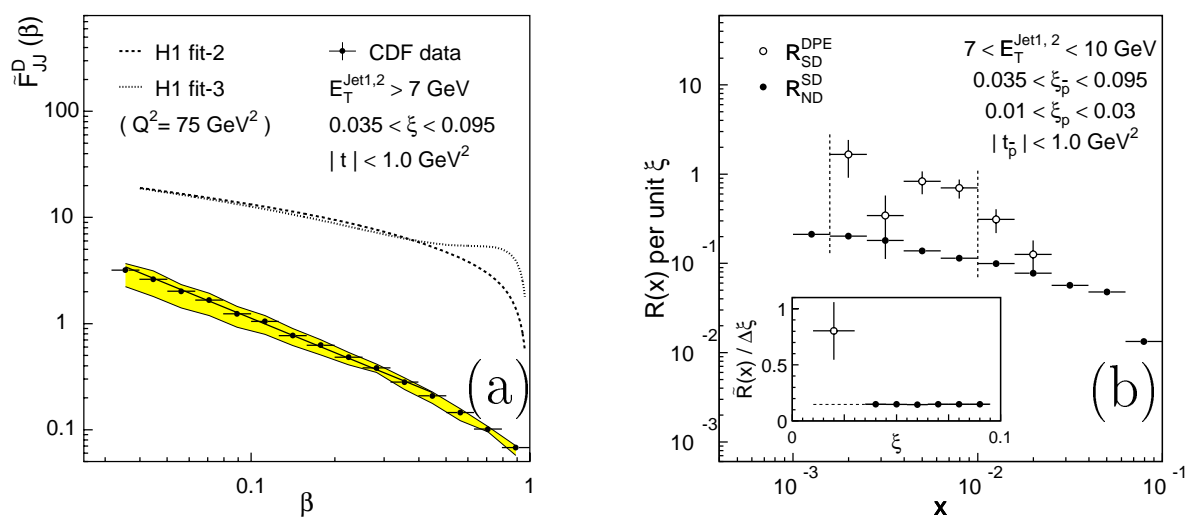

Fig. 2. (a)The diffractive structure function measured by CDF (data points and fit) compared with expectations based on the H1 fit 2 (dashed) and fit 3 (dotted) on diffractive DIS data at HERA (a more recent $\mathrm{H} 1$ fit on a more extensive data set yields a prediction similar in magnitude to that of fit 2 but with a shape which is in agreement with that of the CDF measurement). (b) The ratio of DPE/SD rates compared with that of $\mathrm{SD} / \mathrm{ND}$ rates as a function of $x$-Bjorken of the struck parton in the escaping nucleon. The inequality of the two ratios indicates a breakdown of factorization. 


\section{Double-gap soft diffraction}

The motivation for studying events of the type shown in Fig. 1 is its potential for providing further understanding of the underlying mechanism responsible for the suppression of diffractive cross sections at high energies relative to Regge theory predictions. As shown in Fig. 3, such a suppression has been observed for both single diffraction (SD), $\bar{p}(p)+p \rightarrow[\bar{p}(p)+g a p]+X$, and double diffraction (DD), $\bar{p}(p)+p \rightarrow X_{1}+g a p+X_{2}$.
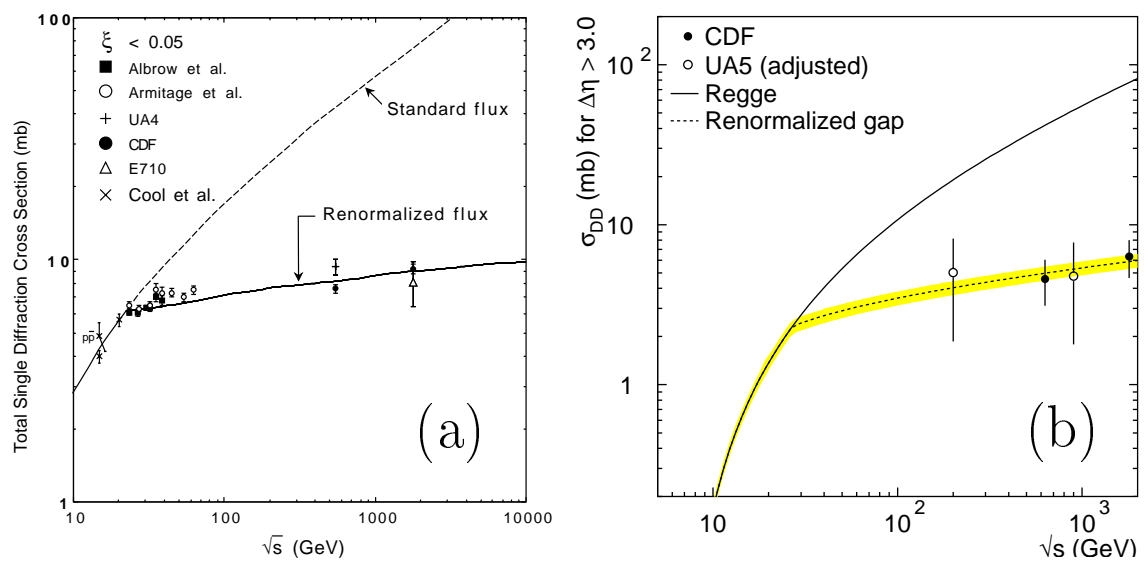

Fig. 3. (a) The $\bar{p} p$ total SD cross section exhibits an $s$-dependence consistent with the renormalization procedure of Ref. [10], contrary to the $s^{2 \epsilon}$ behaviour expected from Regge theory (figure from Ref. [10]); (b) the $\bar{p} p$ total DD (central gap) cross section agrees with the prediction of the renormalized rapidity gap model [11], contrary to the $s^{2 \epsilon}$ expectation from Regge theory (figure from Ref. [12])

Naively, the suppression relative to Regge based predictions is attributed to the spoiling of the diffractive rapidity gap by color exchanges in addition to Pomeron exchange. In an event with two rapidity gaps, additional color exchanges would generally spoil both gaps. Hence, ratios of two-gap to one-gap rates should be unsuppressed. Measurements of such ratios could therefore be used to test the QCD aspects of gap formation without the complications arising from the rapidity gap survival probability.

\section{Data and results}

The data used for this study are inclusive SD event samples at $\sqrt{s}=1800$ and $630 \mathrm{GeV}$ collected by triggering on a leading antiproton detected in a 
Roman Pot Spectrometer (RPS) [5,6]. Below, we list the number of events used in each analysis within the indicated regions of antiproton fractional momentum loss $\xi_{\bar{p}}$ and 4-momentum transfer squared $t$, after applying the vertex cuts $\left|z_{v t x}\right|<60 \mathrm{~cm}$ and $N_{v t x} \leq 1$ and a 4-momentum squared cut of $|t|<0.02 \mathrm{GeV}^{2}$ (except for DPE at $1800 \mathrm{GeV}$ for which $|t|<1.0 \mathrm{GeV}^{2}$ ):

Table 2. Events used in the double-gap analyses

\begin{tabular}{lccc}
\hline Process & $\xi$ & Events at $1800 \mathrm{GeV}$ Events at $630 \mathrm{GeV}$ \\
\hline SDD & $0.06<\xi<0.09$ & $412 \mathrm{~K}$ & $162 \mathrm{~K}$ \\
DPE & $0.035<\xi<0.095$ & $746 \mathrm{~K}$ & $136 \mathrm{~K}$ \\
\hline
\end{tabular}

In the SDD analysis, the mean value of $\xi=0.07$ corresponds to a diffractive mass of $\approx 480(170) \mathrm{GeV}$ at $\sqrt{s}=1800(630) \mathrm{GeV}$. The diffractive cluster $\mathrm{X}$ in such events covers almost the entire CDF calorimetry, which extends through the region $|\eta|<4.2$. Therefore, we use the same method of analysis as that used to extract the gap fraction in the case of DD [12]. We search for experimental gaps overlapping $\eta=0$, defined as regions of $\eta$ with no tracks or calorimeter towers above thresholds chosen to minimize calorimeter noise contributions. The results, corrected for triggering efficiency of $B B C_{p}$ (the beam counter array on the proton side) and converted to nominal gaps defined by $\Delta \eta=\ln \frac{s}{M_{1}^{2} M_{2}^{2}}$, are shown in Fig. 4 .
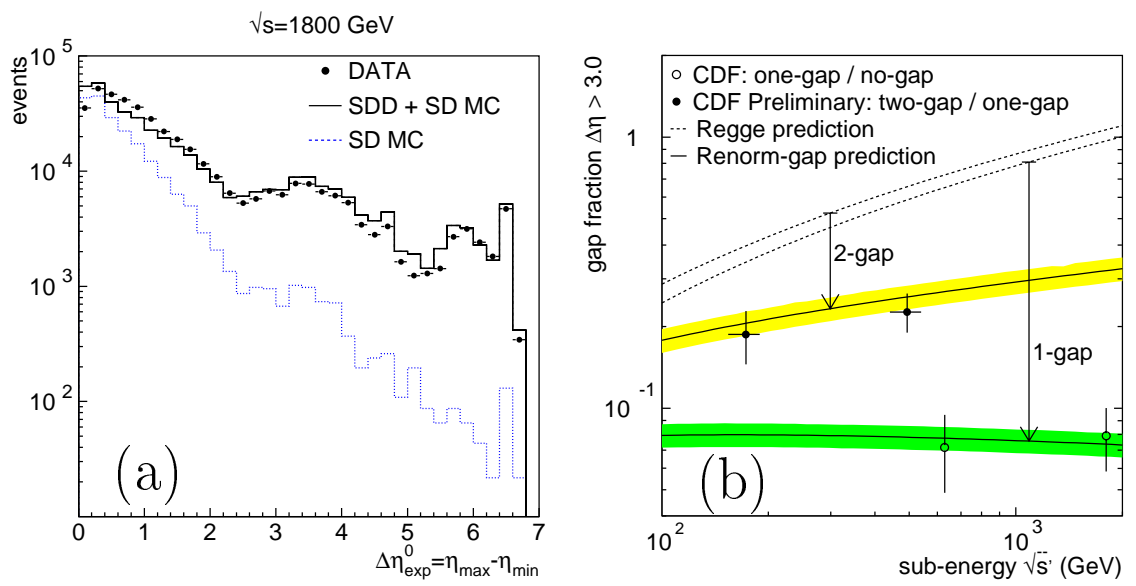

Fig. 4. (a) The number of events as a function of $\Delta \eta_{\exp }^{0}=\eta_{\max }-\eta_{\min }$ for data at $\sqrt{s}=1800 \mathrm{GeV}$ (points), for SDD Monte Carlo generated events (solid line), and for only SD Monte Carlo events (dashed line); (b) ratios of SDD to SD rates (points) and DD to total (no-gap) rates (open circles) as a function of $\sqrt{s^{\prime}}$ of the sub-process $\mathbb{P} p$ and of $\bar{p} p$, respectively. The uncertainties are highly correlated among all data points. 
The SDD Monte carlo simulation is based on Regge theory Pomeron exchange with the normalization left free to be determined from the data. The differential $d N / d \Delta \eta^{0}$ shape agrees with the theory (Fig. 4a), but the two-gap to one-gap ratio is suppressed (Fig. 4b). However, the suppression is not as large as that in the one-gap to no-gap ratio. The bands through the data points represent predictions of the renormalized multigap parton model approach to diffraction [13], which is a generalization of the renormalization models used for single [10] and double [11] diffraction.

In the DPE analysis, the $\xi_{p}$ is measured from calorimeter and beam counter information using the formula below and summing over all particles, defined experimentally as beam-beam counter (BBC) hits or calorimeter towers above $\eta$-dependent thresholds chosen to minimize noise contributions.

$$
\xi_{p}^{\mathrm{X}}=\frac{M_{\mathrm{X}}^{2}}{\xi_{\bar{p}} \cdot s}=\frac{\sum_{i} E_{\mathrm{T}}^{i} \exp \left(+\eta^{i}\right)}{\sqrt{s}}
$$

For BBC hits we use the average value of $\eta$ of the BBC segment of the hit and an $E_{T}$ value randomly chosen from the expected $E_{T}$ distribution. The $\xi^{X}$ obtained by this method was calibrated by comparing $\xi_{\bar{p}}^{X}$, obtained by using $\exp \left(-\eta^{i}\right)$ in the above equation, with the value of $\xi_{\bar{p}}^{R P S}$ measured by the Roman Pot Spectrometer.

Figure 5a shows the $\xi_{\bar{p}}^{X}$ distribution for $\sqrt{s}=1800 \mathrm{GeV}$. The bump at $\xi_{\bar{p}}^{X} \sim 10^{-3}$ is attributed to central calorimeter noise and is reproduced in Monte Carlo simulations. The variation of tower $E_{T}$ threshold across the various components of the CDF calorimetry does not affect appreciably the slope of the $\xi_{\bar{p}}^{X}$ distribution. The solid line represents the distribution measured in SD [14]. The shapes of the DPE and SD distributions are in good agreement all the way down to the lowest values kinematically allowed.

Table 3. Double-gap to single-gap event ratios

\begin{tabular}{lcc}
\hline Source & $R_{S D}^{D P E}(1800 \mathrm{GeV})$ & $R_{S D}^{D P E}(630 \mathrm{GeV})$ \\
\hline Data & $0.197 \pm 0.010$ & $0.168 \pm 0.018$ \\
$P_{\text {gap }}$ renormalization & $0.21 \pm 0.02$ & $0.17 \pm 0.02$ \\
Regge $\oplus$ factorization & $0.36 \pm 0.04$ & $0.25 \pm 0.03$ \\
$\mathbb{P}$-flux renormalization & $0.041 \pm 0.004$ & $0.041 \pm 0.004$ \\
\hline
\end{tabular}

The ratio of DPE to inclusive SD events was evaluated for $\xi_{p}^{X}<0.02$. The results for $\sqrt{s}=1800$ and $630 \mathrm{GeV}$ are presented in Table 4 and shown in Fig. 5b. Also presented in the table are the expectations from gap probability renormalization [13], Regge theory and factorization, and Pomeron flux renormalization for both exchanged Pomerons [10]. The quoted uncertainties are largely systematic for both data and theory; the theoretical uncertainties 
of $10 \%$ are due to the uncertainty in the ratio of the triple-Pomeron to the Pomeron-nucleon couplings [15].

The data are in excellent agreement with the predictions of the gap renormalization approach.
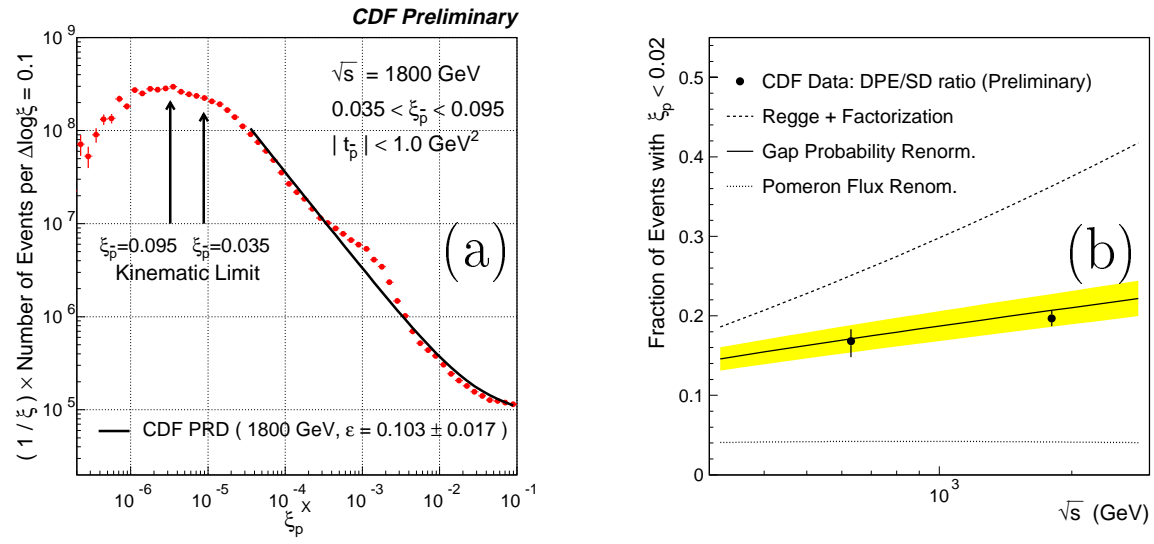

Fig. 5. (a) $\xi_{\bar{p}}^{X}$ distribution at $\sqrt{s}=1800 \mathrm{GeV}$ for events with a $\bar{p}$ of $0.035<\xi_{\bar{p}}^{R P S}<$ 0.095. The solid line is the distribution obtained in single diffraction dissociation. The bump at $\xi_{\bar{p}}^{X} \sim 10^{-3}$ is due to central calorimeter noise and is reproduced in Monte Carlo simulations. (b) Measured ratios of DPE to SD rates (points) compared with predictions based on Regge theory(dashed), Pomeron flux renormalization for both exchanged Pomerons (dotted) and gap probability renormalization (solid line).

\section{References}

1. F. Abe et al., CDF Collaboration: Phys. Rev. Lett. 78, 2698 (1997).

2. F. Abe et al., CDF Collaboration: Phys. Rev. Lett. 79, 2636 (1997).

3. T. Affolder et al., CDF Collaboration: Phys. Rev. Lett. 84, 232 (2000).

4. T. Affolder et al., CDF Collaboration: Phys. Rev. Lett. 87, 241802 (2001)

5. T. Affolder et al., CDF Collaboration: Phys. Rev. Lett. 84, 5083 (2000).

6. D. Acosta et al., CDF Collaboration: Phys. Rev. Lett. 88, 151802 (2002).

7. T. Affolder et al., CDF Collaboration: Phys. Rev. Lett. 85, 4215 (2000).

8. F. Abe et al., CDF Collaboration: Phys. Rev. Lett. 80, 1156 (1998).

9. F. Abe et al., CDF Collaboration: Phys. Rev. Lett. 81, 5278 (1998).

10. K. Goulianos: Phys. Lett. B 358, 379 (1995); B363, 268 (1995).

11. K. Goulianos: arXiv:hep-ph/9806384.

12. T. Affolder et al., CDF Collaboration: Phys. Rev. Lett. 87, 141802 (2001).

13. K. Goulianos: hep-ph/0110240; hep-ph/0203141.

14. F. Abe et al.: Phys. Rev. D 50 (1994) 5535.

15. K. Goulianos and J. Montanha: Phys. Rev. D 50, 114017 (1999). 